\title{
MAYER-VIETORIS PROPERTY OF THE FIXED POINT INDEX.
}

\author{
HÉCTOR BARGE AND KLAUDIUSZ WÓJCIK
}

\begin{abstract}
In this paper we study a Mayer-Vietoris kind of formula for the fixed point index of maps of ENR triplets $f:\left(X ; X_{1}, X_{2}\right) \rightarrow\left(X ; X_{1}, X_{2}\right)$ having compact fixed point set. We prove it under some suitable conditions. For instance when $\left(X ; X_{1}, X_{2}\right)=$ $\left(E^{n} ; E_{+}^{n}, E_{-}^{n}\right)$.

We use these results to generalize Poincaré-Bendixson index formula for vector fields to continuos maps having a sectorial decomposition, to study the fixed point index $i(f, 0)$ of orientation preserving homeomorphisms of $E_{+}^{2}$ and $\left(E^{3} ; E_{+}^{3}, E_{-}^{3}\right)$ and the fixed point index in the invariant subspace.
\end{abstract}

\section{INTRODUCTION}

In this paper we will deal with triplets $\left(X ; X_{1}, X_{2}\right)$ where $X$ is an ENR and $X_{1}, X_{2}$ are ENR's closed in $X$ such that $X_{1} \cap X_{2}$ is also an ENR and $X=X_{1} \cup X_{2}$. We will call such triplets $\left(X ; X_{1}, X_{2}\right)$ ENR triplets and we will denote $X_{0}:=X_{1} \cap X_{2}$. A continuous map $f$ : $\left(X ; X_{1}, X_{2}\right) \rightarrow\left(X ; X_{1}, X_{2}\right)$ of a triplet is a continuous map $f: X \rightarrow X$ satisfying that

$$
f\left(X_{i}\right) \subset X_{i} \quad i=1,2 .
$$

Notice that it follows $f\left(X_{0}\right) \subset X_{0}$.

We denote

$$
f_{i}:=\left.f\right|_{X_{i}}: X_{i} \rightarrow X_{i}, \quad i=0,1,2 .
$$

Let $\operatorname{Fix}(f)=\{x \in X: f(x)=x\}$ be the set of fixed points of $f$. Assuming that Fix $(f)$ is compact it follows that the fixed point indices

$$
i(f, X), \quad \text { and } i\left(f_{i}, X_{i}\right), \quad i=0,1,2,
$$

are well-defined. Our goal is to study the following Mayer-Vietoris index formula

$$
i(f, X)+i\left(f_{0}, X_{0}\right)=i\left(f_{1}, X_{1}\right)+i\left(f_{2}, X_{2}\right)
$$

If $X$ is compact then (0.1) holds by the Lefschetz-Hopf fixed point theorem and the Mayer-Vietoris sequence of the triad $\left(X ; X_{1}, X_{2}\right)$ (see [1, Corollary 2.4]).

The main motivation to study the non-compact case is when

$$
X=E^{n}, \quad X_{+}=E_{+}^{n}, \quad X_{-}=E_{-}^{n},
$$

Where $E^{n}$ denotes the $n$-dimensional Euclidean space and

$$
E_{+}^{n}:=\left\{\left(x_{1}, \ldots, x_{n}\right) \in E^{n}: x_{n} \geq 0\right\} \quad \text { and } \quad E_{-}^{n}:=\left\{\left(x_{1}, \ldots, x_{n}\right) \in E^{n}: x_{n} \leq 0\right\} .
$$

We will denote by $E_{0}^{n}$ to the intersection $E_{+}^{n} \cap E_{-}^{n}$.

The interest of this case comes from applications to ecological systems $([6,11,16,26])$ where $E_{+}^{n}$ is a natural phase space.

Let $M$ be an $n$-dimensional manifold with non-empty boundary $\partial M$. Assume that $f$ : $(M, \partial M) \rightarrow(M, \partial M)$ is a continuous map and let $f_{0}:=\left.f\right|_{\partial M}: \partial M \rightarrow \partial M$. If $p \in \partial M$ is an isolated fixed point of $f$, that is, $f(q) \neq q$ for all $q \neq p$ in a neighborhood of $p$ in $M$, then $p$ is an isolated fixed point of $f_{0}$ as well. A natural problem is to study the relationship between the fixed point indices $i(f, p)$ and $i\left(f_{0}, p\right)$. It follows from [5] that, without further assumptions, the values $i(f, p)$ and $i\left(f_{0}, p\right)$ can be arbitrary integers, with the values unrelated to each other. It can happen even in the smooth category. For instance, if $M=E_{+}^{n}$ then, given integers $r, s \in \mathbb{Z}$, there exists a smooth map $f:\left(E_{+}^{n}, E_{0}^{n}\right) \rightarrow\left(E_{+}^{n}, E_{0}^{n}\right)$ such that the origin 0 is an isolated fixed point of $f$ and

$$
i(f, 0)=r \quad \text { and } \quad i\left(f_{0}, 0\right)=s .
$$

2010 Mathematics Subject Classification. Primary 37C25; Secondary 37B30, 55M25

Key words and phrases. fixed point index, Brouwer degree, sectorial decomposition, proper pair, isolated invariant set. 
However, if $f:(M, \partial M) \rightarrow(M, \partial M)$ is smooth, $p \in \partial M$ is an isolated fixed point of $f$ and $f^{\prime}(p)-I: T_{p}(\partial M) \rightarrow T_{p}(\partial M)$ is a nonsingular linear transformation then either $i(f, p)=0$ or $i(f, p)=i\left(f_{0}, p\right)= \pm 1($ see $[5,20,21])$.

The study of the important case $M=E_{+}^{n}$ is mostly motivated by ecological systems (compare $[6,11,16,26,33,34])$ and is closely related to permanence theory, where, roughly speaking, conditions for $E_{0}^{n}$ to be a repeller are studied. From the point of view of biological applications the fact that $\emptyset \neq \omega(x) \subset E_{0}^{n}$ for some $x \in E_{+}^{n} \backslash E_{0}^{n}$, is understood as a strong violation of permanence (the ultimate survival of all spieces).

In this context, a special kind of compact invariant sets $S \subset E_{0}^{n}$, the so-called sets of repelling type (see $[11,33,34])$ are interesting. It turns out that it is common in biological applications that $S$ is an isolated invariant set in the sense of the Conley index theory (i.e., isolated with respect to the entire flow or map on $\left.E_{+}^{n}\right)$. For instance, in [16] Hofbauer initiated the study of $S$ being a finite collection of equilibria by means of standard degree theory. Capietto and Garay [6] used the fixed point index theory and the Conley index theory. Their approach, hovewer, only worked for flows induced by vector fields and an special kind of isolated invariant sets called saturated invariant sets. Both restrictions were removed and much more Conley-type results were proved in [33, 34]. Generalizations for discrete-time semidynamical systems were presented in [31].

For instance, if $S \subset E_{0}^{n}$ is an isolated invariant set of a continuous map $f:\left(E_{+}^{n}, E_{0}^{n}\right) \rightarrow$ $\left(E_{+}^{n}, E_{0}^{n}\right)$ it follows from the results in [31] that

$$
i(f, S)=i\left(f_{0}, S\right),
$$

provided $W^{u}(S) \subset E_{0}^{n}$ and

$$
i(f, S)=0,
$$

if $W^{s}(S) \subset E_{0}^{n}$. In particular, if $\{0\}$ is an isolated invariant set of $f$ then

$$
i(f, 0)= \begin{cases}i\left(f_{0}, 0\right) & \text { if } W^{u}(0) \subset E_{0}^{n} \\ 0 & \text { if } W^{s}(0) \subset E_{0}^{n} .\end{cases}
$$

Let us mention, that it follows from the construction in [29] that for $n \leq 1, k \in \mathbb{Z}$ there exists a homeomorphism $f: E_{+}^{3} \rightarrow E_{+}^{3}$ such that $\{0\}$ is an isolated invariant set and

$$
i(f, 0)=k, \quad i\left(f_{0}, 0\right)=n .
$$

The paper is structured as follows:

In Section 1 the notion of trapping set is introduced and it is proven that if $A$ is a trapping set and $K=\operatorname{Fix}\left(\left.f\right|_{A}\right)$ is compact, then $i(f, K)$ is well-defined and agrees with $i\left(\left.f\right|_{A}, K\right)$. This result plays a key role in the proof of Proposition 5, which is the main result of this section and establishes that if $f:\left(X ; X_{1}, X_{2}\right) \rightarrow\left(X ; X_{1}, X_{2}\right)$ is a continuous map whose fixed is compact and $X_{0}$ is a trapping set, then $(0.1)$ holds.

In Section 2 we recall the definition and properties of proper pairs introduced by Srzednicki in [30]. In addition, we introduce the concept of admissible proper pair with respect to a map $f$ : $\left(X ; X_{1}, X_{2}\right) \rightarrow\left(X ; X_{1}, X_{2}\right)$ and we prove Theorem 9, which establishes that if $\operatorname{Fix}(f) \subset \operatorname{int}(C \backslash E)$ for some admissible proper pair $(C, E)$ with respect to $f$, then $(0.1)$ holds.

In Section 3 we study the Brouwer degree of maps $f:\left(S^{n}, S^{n-1}\right) \rightarrow\left(S^{n}, S^{n-1}\right), n \geq 1$. The main result of this section is Theorem 12 which establishes that if $f_{+}:=\left.f\right|_{S_{+}^{n}}$ and $f_{-}:=\left.f\right|_{S_{-}^{n}}$ admit continuous extensions $F_{1}$ and $F_{2}$ respectively, satisfying

$$
F_{1}\left(S_{-}^{n} \backslash S^{n-1}\right) \subset S_{-}^{n} \backslash S^{n-1} \text { and } F_{2}\left(S_{+}^{n} \backslash S^{n-1}\right) \subset S_{+}^{n} \backslash S^{n-1},
$$

then

where $f_{0}:=\left.f\right|_{S^{n-1}}: S^{n-1} \rightarrow S^{n-1}$.

$$
\operatorname{deg}(f)+\operatorname{deg}\left(f_{0}\right)=\operatorname{deg}\left(F_{1}\right)+\operatorname{deg}\left(F_{2}\right),
$$

As a consequence of this result we obtain Proposition 13 which establishes that if $f: S^{n} \rightarrow S^{n}$ is symmetric with respect to $S^{n-1}$, then $f\left(S^{n-1}\right) \subset S^{n-1}$ and $\operatorname{deg}\left(f_{0}\right)$ is congruent to $\operatorname{deg}(f)$ modulo 2 .

In Section 4 we prove, using the results of Section 3, Theorem 14, which establishes that if $f:\left(E^{n} ; E_{+}^{n}, E_{-}^{n}\right) \rightarrow\left(E^{n} ; E_{+}^{n}, E_{-}^{n}\right)$ is a continuous map whose fixed point set is compact, then (0.1) holds. In addition, we introduce the concept of good Euclidean embedding and we see that if $f:\left(X ; X_{1}, X_{2}\right) \rightarrow\left(X ; X_{1}, X_{2}\right)$ is a continuous map of a triplet which admits a good Euclidean embedding and $\operatorname{Fix}(f)$ is compact, then $(0.1)$ holds.

Section 5 is devoted to applications of the results of Section 4. In particular we prove Lemma 23 and Corollary 25 which generalize to the context of continuous maps $f: E^{2} \rightarrow E^{2}$ the classical Poincaré-Bendixson index formula for vector fields. In addition, we obtain some consequences of 
these results. For instance, we see that if $f: E_{+}^{2} \rightarrow E_{+}^{2}$ is an orientation preserving homeomorphism having 0 as its only fixed point, the sequence $\left(i\left(f^{n}, 0\right)\right)_{n>0}$ is well-defined and constant and we obtain some upper-bounds to $i(f, 0)$ if $f$ is also area preserving.

In Section 6 we recall the concept isolated invariant set of a map and we prove Proposition 30, which is an application of Theorem 14 to isolated invariant sets in the invariant subspace.

In Section 7 we study some applications of Theorem 14 to homeomorphisms $f:\left(E^{3} ; E_{+}^{3}, E_{-}^{3}\right) \rightarrow$ $\left(E^{3} ; E_{+}^{3}, E_{-}^{3}\right)$ which are symmetric with respecto to $E_{0}^{3}$ and have the origin as an isolated fixed point.

Finally, in Section 8, we present some open problems.

\section{PRELIMINARIES}

Through the paper we will use some algebraic and differential topology techniques. We recommend to the reader the book of Spanier [32] for questions about algebraic topology and [15, 23, 24] as references for differential topology. Regarding the fixed point index theory we recommend [18]. For the sake of completeness we will recall the basic definitions of the degree of maps between smooth manifolds following [24] and for the fixed point index theory we will follow the exposition presented in [19].

Degree of maps between manifolds. Let $M$ and $N$ be smooth orientable manifolds and assume that $M$ is compact and $N$ is connected. Let $f: M \rightarrow N$ be a smooth map. Given $x \in M$, we will denote $f^{\prime}(x)$ to the differential of the map $f$ in $x$. A point $x \in M$ is said regular if $f^{\prime}(x)$ is non-singular. Let $y \in N, y$ is said to be a regular value if $f^{-1}(y)$ only contains regular points. Notice that from Sard's Theorem, the set of regular values is dense in $N$. Besides, as a consequence of the inverse function Theorem, if $y$ is a regular value, $f^{-1}(y)$ consists in a finite number of points. The Brouwer degree of the map $f$ is defined to be

$$
\operatorname{deg}(f)=\sum_{x \in f^{-1}(y)} \operatorname{sgn}\left(\operatorname{det} f^{\prime}(x)\right) .
$$

Where $y$ is a regular value and $\operatorname{sgn}\left(\operatorname{det} f^{\prime}(x)\right)$ denotes the sign of the determinant of the Jacobian matrix of $f^{\prime}(x)$. Notice that the definition does not depend on the choice of the regular value. Moreover, $\operatorname{deg}(f)$ is an invariant of the smooth homotopy class of $f$.

The definition of the degree of a smooth map can be extended to continuous maps in the following way. If $f: M \rightarrow N$ is a continuous map there exists a smooth map $g: M \rightarrow N$ homotopic to $f$ and the Brouwer degree of $f$ is defined as $\operatorname{deg}(f):=\operatorname{deg}(g)$.

Notice that the degree of continuous maps is a well-defined integer number and it is an invariant of the homotopy class of the map. This follows from the fact that if two smooth maps between smooth manifolds are homotopic they must be smoothly homotopic.

Let $S^{0}=\{-1,1\}$, i.e., the 0 -dimensional sphere. For technical reasons we also have to define the degree of a map $f: S^{0} \rightarrow S^{0}$. In this case any point in $S^{0}$ is going to be considered a regular value and the degree is defined as follows:

$$
\operatorname{deg}(f)=\sum_{x \in f^{-1}(y)} \operatorname{sgn}\left(\operatorname{det} f^{\prime}(x)\right),
$$

where $y$ is any point in $S^{0}$ and

$$
\operatorname{sgn}\left(\operatorname{det} f^{\prime}(x)\right):= \begin{cases}-1 & \text { if } f(x) \neq x \\ 1 & \text { if } f(x)=x .\end{cases}
$$

A direct consequence of the definition is that any map $f: S^{0} \rightarrow S^{0}$ has degree either $-1,0$ or 1 . Notice that here we use the notation $\operatorname{sgn}\left(\operatorname{det} f^{\prime}(x)\right)$ to be in accordance with the higher-dimensional case previously described but it does not have any meaning in connection with differential topology.

More generally, if $J=[a, b]$ is an interval of the real line and $f: \partial J \rightarrow S^{0}$ is a map, the degree of $f$ is defined as

$$
\operatorname{deg}(f)=\operatorname{deg}(f \circ h)
$$

where $h: S^{0} \rightarrow \partial J$ is the map which identifies $S^{0}$ with $\partial J$ preserving the natural order induced by the real line in $S^{0}$ and $\partial J$, i.e., such that $h(-1)=a$ and $h(1)=b$.

Fixed point index on the Euclidean space. Let $U$ be an open subset of $E^{n}$ and $f: U \rightarrow$ $E^{n}$ a continuous map such that $\operatorname{Fix}(f)$ is compact. Choose a compact neighborhood $V \subset U$ of Fix $(f)$ which is a compact smooth $n$-manifold with boundary. Define $\Phi: \partial V \rightarrow S^{n-1}$ as

The fixed point index of $f$ in $U$ is defined as

$$
\Phi(x)=\frac{x-f(x)}{\|x-f(x)\|} .
$$

$$
i(f, U)=\operatorname{deg}(\Phi) .
$$


Notice that, $i(f, U)$ is an integer number, that it does not depend on the choice of $V$ and that it is an invariant of the homotopy class of $f$. Notice also that in the case $n=1, V$ could be a disjoint union of closed intervals $I_{1}, \ldots, I_{k}$. In this case we would define,

$$
\operatorname{deg}(\Phi):=\sum_{i=1}^{k} \operatorname{deg}\left(\left.\Phi\right|_{\partial I_{i}}\right) .
$$

Fixed point index on ENR's. Let $X$ be an ENR and $f: X \rightarrow X$ is a continuous map satisfying that $\operatorname{Fix}(f)$ is compact. Since $X$ is an ENR, it can be embedded in some $E^{n}$ in such a way that if $e: X \rightarrow E^{n}$ is an embedding, there exists an open set $W \subset E^{n}$ such that $e(X) \subset W$ and a retraction $r: W \rightarrow e(X)$. Consider the map $F=e \circ f \circ r$. The fixed point index of $f$ in $X$ is defined as

$$
i(f, X):=i(F, W) .
$$

The definition does not depend on the choice of $e, W$ and $r$. Following this construction, if $f$ is only defined in an open subset $U$ of $X$, the index is defined as

$$
i(f, U):=i\left(\left.F\right|_{r^{-1}(U)}, r^{-1}(U)\right) .
$$

Suppose that $X$ is a compact ENR and $H_{*}$ is the singular homology functor with rational coefficients. Then $H_{*}(X)$ is of finite type, i.e., $H_{n}(X)$ is finite dimensional for all $n$, and zero if $n$ is greater than a certain $n_{0}$. If $f: X \rightarrow X$ is a continuous map, then the Lefschetz number of $f$ is defined as

$$
L(f)=\sum_{n=1}^{\infty}(-1)^{n} \operatorname{tr}\left(f_{* n}\right),
$$

where $f_{* n}: H_{n}(X) \rightarrow H_{n}(X)$ is the linear map induced from $f$.

It can be proved that $L(f)$ is an integer and it is obviously an invariant of the homotopy class of $f$.

The Lefschetz number and the fixed points index of a continuous self-map $f: X \rightarrow X$ of a compact ENR are related through the celebrated Lefschetz-Hopf Theorem (see [8]).

Theorem 1. Let $f: X \rightarrow X$ be a continuous self-map of a compact ENR. Then, $L(f)=i(f, X)$.

Isolated sets of fixed points. Consider a continuous map $f: U \rightarrow X$, where $X$ is a compact ENR and assume that $\operatorname{Fix}(f)$ is compact. We will say that a closed subset $K \subset \operatorname{Fix}(f)$ is an isolated set of fixed points if there exists an open subset $U^{\prime}$ of $U$ such that $U^{\prime} \cap \operatorname{Fix}(f)=K$. In particular, if $K$ is a singleton $\left\{x_{0}\right\}$ we will say that $x_{0}$ is an isolated fixed point.

If $K$ is an isolated set of fixed points we will denote $i(f, K):=i\left(\left.f\right|_{U^{\prime}}, U^{\prime}\right)$, where $U^{\prime}$ is any open subset such that $U^{\prime} \cap \operatorname{Fix}(f)=K$. As a consequence the localization property of the fixed point index this is well-defined. Notice that in general, if $U^{\prime}$ is an open subset of the domain of $f$ such that $U^{\prime} \cap \operatorname{Fix}(f)$ is compact we will denote $i\left(f, U^{\prime}\right):=i\left(\left.f\right|_{U^{\prime}}, U^{\prime}\right)$.

\section{Trapping Sets and the Mayer-Vietoris indeX formula}

In this section we will introduce the concept of trapping set of a map and we will see that if $\left(X ; X_{1}, X_{2}\right)$ is an ENR triplet, $f:\left(X ; X_{1}, X_{2}\right) \rightarrow\left(X ; X_{1}, X_{2}\right)$ is a continuous map having a compact fixed point set and $X_{0}$ is a trapping set, then (0.1) holds.

Definition 2. Let $f: X \rightarrow X$ be a continuous map and $A$ a closed subset of $X$. We say that $A$ is a trapping set of $f$ if there exists an open neighborhood $U$ of $A$ in $X$ such that $f(U) \subset A$.

Remark 3. Notice that if a closed subset $A$ of $X$ is a trapping set of a continuous map $f: X \rightarrow X$, then $f(A) \subset A$.

Lemma 4. Assume that $(X, A)$ is a pair of ENR's and $A \subset X$ is closed. Let $f: X \rightarrow X$ be a continuous map such that $\operatorname{Fix}(f)$ is compact. Suppose that $A$ is a trapping set of $f$, then $K=\operatorname{Fix}\left(\left.f\right|_{A}\right)$ is an isolated set of fixed points and

$$
i(f, K)=i\left(\left.f\right|_{A}, A\right) .
$$

Proof. Since $K$ agrees with $\operatorname{Fix}(f) \cap A$, it is compact. Moreover, if $U$ is an open neighborhood of $A$ trapped by $A$ (i.e. $f(U) \subset A$ ), then $K=\operatorname{Fix}(f) \cap U$. As a consequence, $K$ is an isolated set of fixed points of $f$. It follows from the commutativity of the fixed point index that $i(f, K)=$ $i\left(\left.f\right|_{A}, K\right)=i\left(\left.f\right|_{A}, A\right)$.

Proposition 5. Let $\left(X ; X_{1}, X_{2}\right)$ be an ENR triplet and $f:\left(X ; X_{1}, X_{2}\right) \rightarrow\left(X ; X_{1}, X_{2}\right)$ a continuous map having a compact fixed point set. If $X_{0}$ is a trapping set of $f$, then (0.1) holds. 
Proof. Let $U$ be an open neighborhood of $X_{0}$ trapped by $X_{0}$. Then, Lemma 4 ensures that $\operatorname{Fix}(f)$ is the disjoint union of the compact sets

$$
K_{0}=\operatorname{Fix}(f) \cap X_{0} \quad \text { and } \quad K_{i}=\operatorname{Fix}(f) \cap\left(X_{i} \backslash X_{0}\right), \quad i=1,2 .
$$

As a consquence, the additivity property of the fixed point index ensures that

$$
i(f, X)=\sum_{i=0}^{3} i\left(f, K_{i}\right)
$$

Reasoning in an analogous way with $X_{i}, i=1,2$ we get

$$
i\left(f_{i}, X_{i}\right)=i\left(f_{i}, K_{i}\right)+i\left(f_{i}, K_{0}\right),
$$

On the other hand, from the localization property of the fixed point index it follows that

$$
i\left(f, K_{i}\right)=i\left(f_{i}, K_{i}\right), \quad i=1,2
$$

Finally, from Lemma 4 we obtain that for $i=1,2$

$$
i\left(f, K_{0}\right)=i\left(f_{i}, K_{0}\right)=i\left(f_{0}, K_{0}\right)=i\left(f_{0}, X_{0}\right) .
$$

The result is straightforward from (1.1), (1.2), (1.3) and (1.4).

2. Proper pairs And Mayer-Vietoris index formula

In this section we will study (0.1) using the concept of proper pair introduced by Srzednicki in [30]. In particular we will see that if $\left(X ; X_{1}, X_{2}\right)$ is an ENR triplet and $f:\left(X ; X_{1}, X_{2}\right) \rightarrow$ $\left(X ; X_{1}, X_{2}\right)$ is a continuous map such that $\operatorname{Fix}(f) \subset \operatorname{int}(C \backslash E)$, where $(C, E)$ is what we call an admissible proper pair with respect to $f$, then $(0.1)$ holds.

Let $C$ and $E$ be compact subsets of $X, E \subset C$. Following [30], we say that the pair $(C, E)$ is proper with respect to a continuous map $f: X \rightarrow X$ if it satisfies that

$$
C \cap f(E) \subset E, \quad C \cap \overline{f(C) \backslash C} \subset E .
$$

Remark 6. Notice that if $A \subset X$ is a closed subset such that $f(A) \subset A$, then the pair $(C \cap A, E \cap A)$ is proper with respect to $\left.f\right|_{A}: A \rightarrow A$.

For a compact pair $(C, E)$ contained in $X$, the following conditions are equivalent

(a) $(C, E)$ is proper with respect to $f$;

(b) There exists a compact pair $\left(C^{\prime}, E^{\prime}\right)$ in $X$ such that:

$$
(C, E) \subset\left(C^{\prime}, E^{\prime}\right), \quad f(C) \subset C^{\prime}, f(E) \subset E^{\prime}, \quad C \backslash E=C^{\prime} \backslash E^{\prime} .
$$

(c) $(C, E)$ satisfies $C \cap f(E) \subset E$ and the map $f^{\dagger}: C / E \rightarrow C / E$ given by

is continuous.

$$
f^{\dagger}([x])=\left\{\begin{array}{ll}
{[f(x)],} & \text { if } f(x) \in C \backslash E \\
\star, & \text { otherwise }
\end{array} \quad \text { with } \star=[E],\right.
$$

Let $H^{*}$ be the Alexander-Spanier cohomology functor with rational coefficients. For $\left(C^{\prime}, E^{\prime}\right)$ we define the transfer homomorphism $f^{\sharp}:=f_{(C, E)}^{\sharp}: H^{*}(C, E) \rightarrow H^{*}(C, E)$ as the composition

$$
f^{\sharp}: H^{*}(C, E) \stackrel{\cong}{\longrightarrow} H^{*}\left(C^{\prime}, E^{\prime}\right) \stackrel{f^{*}}{\longrightarrow} H^{*}(C, E),
$$

where the first arrow is the inverse of the isomorphism induced by the inclusion $(C, E) \hookrightarrow\left(C^{\prime}, E^{\prime}\right)$ (we use the strong excision property of the Alexander-Spanier cohomology). The definition of the transfer homomorphism does not depend on the choice $\left(C^{\prime}, E^{\prime}\right)$ in $b$.

Theorem 7 (Srzednicki). Assume that $X$ is an ENR. If $(C, E)$ is a pair of compact ENR's in $X$, proper with respect to $f$ and

$$
\operatorname{Fix}(f) \cap \overline{C \backslash E} \subset \operatorname{int}(C \backslash E)
$$

then

$$
i(f, \operatorname{int}(C \backslash E))=L\left(f^{\sharp}\right) .
$$

Let $\left(X ; X_{1}, X_{2}\right)$ be an ENR triplet and $f:\left(X ; X_{1}, X_{2}\right) \rightarrow\left(X: X_{1}, X_{2}\right)$ a continuous map. Let $(C, E)$ be a proper pair with respect to $f$. We denote by

$$
\left(C_{i}, E_{i}\right)=\left(C \cap X_{i}, E \cap X_{i}\right) \quad i=0,1,2 .
$$

Definition 8. We say that a proper pair $(C, E)$ is admissible if $(C, E)$, and $\left(C_{i}, E_{i}\right)$ are pairs of compact ENR's for $i=0,1,2$. 
Theorem 9. Let $\left(X ; X_{1}, X_{2}\right)$ be an ENR triplet and $f:\left(X ; X_{1}, X_{2}\right) \rightarrow\left(X ; X_{1}, X_{2}\right)$ a continuous map. Suppose that there exists and admissible proper pair $(C, E)$ with respect to $f$ such that $\operatorname{Fix}(f) \subset \operatorname{int}(C \backslash E)$. Then (0.1) holds.

Proof. Notice that, since $\operatorname{Fix}(f)$ is a closed subset of the compact set $C$, it must be compact. It follows from Remark 6 that $\left(C_{i}, E_{i}\right)$ is a proper pair with respect to $f_{i}, i=0,1,2$. Moreover,

$$
\operatorname{Fix}\left(f_{i}\right) \subset \operatorname{int}\left(C_{i} \backslash E_{i}\right), \quad i=0,1,2 .
$$

The following diagram, whose rows are the Mayer-Vietoris sequences of the pairs of excisive triads $\left(E ; E_{1}, E_{2}\right) \subset\left(C ; C_{1}, C_{2}\right)$ is commutative and, since $(C, E)$ is an admissible proper pair, all its entries are finite-dimensional vector spaces and only a finite number of them are non-trivial.

$$
\begin{aligned}
& \cdots \longrightarrow H^{i}(C, E) \longrightarrow H^{i}\left(C_{1}, E_{1}\right) \oplus H^{i}\left(C_{2}, E_{2}\right) \longrightarrow H^{i}\left(C_{0}, E_{0}\right) \longrightarrow f_{\left(C_{0}, E_{0}\right)} \longrightarrow \\
& \quad f_{(C, E)}^{\sharp} \downarrow \\
& \left.\cdots \longrightarrow H^{i}\left(C_{0}, E_{0}\right) \longrightarrow E_{1}\right) \oplus f_{\left(C_{2}, E_{2}\right) \downarrow}^{\longrightarrow} \longrightarrow \cdots
\end{aligned}
$$

As a consequence, from $[1$, Theorem 2.2] we get that

$$
L\left(f_{(C, E)}^{\sharp}\right)+L\left(f_{\left(C_{0}, E_{0}\right)}^{\sharp}\right)=L\left(f_{\left(C_{1}, E_{1}\right)}^{\sharp}\right)+L\left(f_{\left(C_{2}, E_{2}\right)}^{\sharp}\right),
$$

and, hence, Theorem 7 ensures that

$$
i(f, \operatorname{int}(C \backslash E))+\left(f_{0}, \operatorname{int}\left(C_{0} \backslash E_{0}\right)\right)=\left(f_{1}, \operatorname{int}\left(C_{1} \backslash E_{1}\right)\right)+\left(f_{2}, \operatorname{int}\left(C_{2} \backslash E_{2}\right)\right) .
$$

The result follows from (2.1) and the localization property of the index.

\section{A DEGREE FORMUla FOr SPHERE MAPS}

This section is devoted to prove a formula for the degree of maps $f:\left(S^{n}, S^{n-1}\right) \rightarrow\left(S^{n}, S^{n-1}\right)$, $n \geq 1$, which will play a fundamental role in the proof of (0.1) in the Euclidean space.

By $S^{n}$ we mean the unit sphere centered at the origin of the Euclidean space $E^{n+1}$. We are going to denote

$$
S_{+}^{n}:=S^{n} \cap E_{+}^{n+1} \quad \text { and } \quad S_{-}^{n}:=S^{n} \cap E_{-}^{n+1} .
$$

The intersection $S_{+}^{n} \cap S_{-}^{n}$ is exactly the unit sphere $S^{n-1}$ of the Euclidean space $E_{0}^{n+1}=E^{n} \times\{0\}$.

Consider $S^{n-1} \subset E^{n} \times\{0\}$ and $J \subset[-1,1]$. We use the notation

$$
S_{J}:=S^{n-1} \times J \subset E^{n+1} .
$$

Consider the projection $\pi: S_{[-1,1]} \rightarrow S^{n}$ from the cylinder $S_{[-1,1]}$ onto the sphere $S^{n}$ which sends each $(p, t) \in S_{[-1,1]}$ to the point in $S^{n}$ lying in the straight line joining $(p, t)$ with the point $(0, \ldots, 0, t) \in E^{n+1}$.

In particular, we have that

$$
S_{+}^{n}=\pi\left(S_{[0,1]}\right), \quad S_{-}^{n}=\pi\left(S_{[-1,0]}\right),
$$

and, if $J \subset(-1,1)$ is a subinterval, the map $\left.\pi\right|_{S_{J}}: S_{J} \rightarrow \pi\left(S_{J}\right)$ is a diffeomorphism.

Let $f:\left(S^{n}, S^{n-1}\right) \rightarrow\left(S^{n}, S^{n-1}\right)$ be a continuous map. We denote

$$
f_{+}:=\left.f\right|_{S_{+}^{n}}, f_{-}:=\left.f\right|_{S_{-}^{n}} \text { and } f_{0}:=\left.f\right|_{S^{n-1}}: S^{n-1} \rightarrow S^{n-1} .
$$

To prove the formula about the degree we will make use of the following Lemma.

Lemma 10. Let $f:\left(S_{+}^{n}, S^{n-1}\right) \rightarrow\left(S^{n}, S^{n-1}\right)$ be a continuous map. Then, there exists a homotopy $H_{t}:\left(S_{+}^{n}, S^{n-1}\right) \rightarrow\left(S^{n}, S^{n-1}\right)$ such that $H_{0}=f$ and $H_{1}$ is smooth. Moreover, if $f\left(S_{+}^{n}\right) \subset S_{+}^{n}$ then $H$ can be chosen such that $H_{t}\left(S_{+}^{n}\right) \subset S_{+}^{n}$.

Proof. It follows from [15, Theorem 3.5] that $f$ can be arbitrarily close approximated by a smooth map $g:\left(S_{+}^{n}, S^{n-1}\right) \rightarrow\left(S^{n}, S^{n-1}\right)$. Moreover, if $f\left(S_{+}^{n}\right) \subset S_{+}^{n}$ then $g$ can be chosen such that $g\left(S_{+}^{n}\right) \subset S_{+}^{n}$. Let $W$ be an $\epsilon$-neighborhood of $S^{n}$ in $E^{n+1}$ and let $r: W \rightarrow S^{n}$ be a radial retraction. We define $h_{t}: E^{n+1} \rightarrow E^{n+1}$ by

$$
h_{t}(x)=(1-t) f(x)+t g(x) .
$$

We can choose $g$ in such a way that it approximates $f$ so closely that $h_{t}(x) \in W$ for each $x \in S^{n}$ and $t \in[0,1]$. The desired homotopy is $H_{t}:=r \circ h_{t}$. Notice that $H_{t}\left(S^{n-1}\right) \subset S^{n-1}$ since $h_{t}(x) \in E^{n} \times\{0\}$ for every $x \in S^{n-1}$ and $t \in[0,1]$.

The following Lemma states a smooth version of the formula we are looking for. 
Lemma 11. Let $f:\left(S^{n}, S^{n-1}\right) \rightarrow\left(S^{n}, S^{n-1}\right)$ be a smooth map. Suppose that $f_{+}$and $f_{-}$admit smooth extensions $F_{1}$ and $F_{2}$ respectively, satisfying

$$
F_{1}\left(S_{-}^{n} \backslash S^{n-1}\right) \subset S_{-}^{n} \backslash S^{n-1} \quad \text { and } \quad F_{2}\left(S_{+}^{n} \backslash S^{n-1}\right) \subset S_{+}^{n} \backslash S^{n-1},
$$

then

$$
\operatorname{deg}(f)+\operatorname{deg}\left(f_{0}\right)=\operatorname{deg}\left(F_{1}\right)+\operatorname{deg}\left(F_{2}\right) .
$$

Proof. Let $g:\left(S^{n}, S^{n-1}\right) \rightarrow\left(S^{n}, S^{n-1}\right)$ be a continuous map satisfying that if $x \in \pi\left(S_{[-1 / 2,1 / 2]}\right)$,

$$
g(x)=\pi\left(g_{0}(p), t\right),
$$

where $(p, t)$ is the only point in $S_{[-1 / 2,1 / 2]}$ such that $x=\pi(p, t)$.

Then, by Sard's Theorem we have that there exists $x_{0}=\pi\left(p_{0}, t_{0}\right) \in \pi\left(S_{[-1 / 2,1 / 2]}\right)$ regular value of $g$. Then, $g^{-1}\left(x_{0}\right)$ consists of a finite number of points and

$$
\operatorname{deg}(g)=\sum_{x \in g^{-1}\left(x_{0}\right)} \operatorname{sgn}\left(\operatorname{det} g^{\prime}(x)\right)
$$

On the other hand let

$U=g^{-1}\left(x_{0}\right) \cap \pi\left(S_{[-1 / 2,1 / 2]}\right), V=g^{-1}\left(x_{0}\right) \cap \pi\left(S_{(1 / 2,1]}\right) \quad$ and $\quad W=g^{-1}\left(x_{0}\right) \cap \pi\left(S_{[-1,-1 / 2)}\right)$.

Hence,

$$
\operatorname{deg}(g)=\sum_{x \in U} \operatorname{sgn}\left(\operatorname{det} g^{\prime}(x)\right)+\sum_{x \in V} \operatorname{sgn}\left(\operatorname{det} g^{\prime}(x)\right)+\sum_{x \in W} \operatorname{sgn}\left(\operatorname{det} g^{\prime}(x)\right) .
$$

In addition, (3.3) ensures that $p_{0}$ is also a regular value for $g_{0}$, that $\pi\left(g_{0}^{-1}\left(p_{0}\right) \times\left\{t_{0}\right\}\right)$ agrees with $U$ and that for $x=\pi\left(p, t_{0}\right) \in U$

Therefore,

$$
\operatorname{sgn}\left(\operatorname{det} g^{\prime}(x)\right)=\operatorname{sgn}\left(\operatorname{det} g_{0}^{\prime}(p)\right) .
$$

$$
\operatorname{deg}\left(g_{0}\right)=\sum_{x \in U} \operatorname{sgn}\left(\operatorname{det} g^{\prime}(x)\right)
$$

and we get that

$$
\operatorname{deg}(g)=\operatorname{deg}\left(g_{0}\right)+\sum_{x \in V} \operatorname{sgn}\left(\operatorname{det} g^{\prime}(x)\right)+\sum_{x \in W} \operatorname{sgn}\left(\operatorname{det} g^{\prime}(x)\right) .
$$

Notice that we can find smooth maps $\tilde{f}, \tilde{F}_{1}$ and $\tilde{F}_{2}$ homotopic to $f, F_{1}$, and $F_{2}$ relative to $S^{n-1}$ respectively and such that $\tilde{f}$ satisfies (3.3),

$$
\left.\tilde{F}_{1}\right|_{\pi\left(S_{[-1 / 2,1]}\right)}=\left.\tilde{f}\right|_{\pi\left(S_{[-1 / 2,1]}\right)},\left.\quad \tilde{F}_{2}\right|_{\pi\left(S_{[-1,1 / 2]}\right)}=\left.\tilde{f}\right|_{\pi\left(S_{[-1,1 / 2]}\right)}
$$

and

$$
\tilde{F}_{1}\left(\pi\left(S_{[-1,-1 / 2)}\right)\right) \subset \pi\left(S_{[-1,-1 / 2)}\right), \quad \tilde{F}_{2}\left(\pi\left(S_{(1 / 2,1]}\right)\right) \subset \pi\left(S_{(1 / 2,1]}\right) .
$$

Now, $\tilde{f}, \tilde{F}_{1}$ and $\tilde{F}_{2}$ satisfy (3.3) and, hence, if $x_{0} \in \pi\left(S_{[-1 / 2,1 / 2]}\right)$ is a common regular value of the three maps, which exists as a consequence of Sard's Theorem, then from (3.4), (3.5) and (3.6) it follows that

$$
\begin{gathered}
\operatorname{deg}(\tilde{f})=\operatorname{deg}\left(\tilde{f}_{0}\right)+\sum_{x \in V} \operatorname{sgn}\left(\operatorname{det} \tilde{f}^{\prime}(x)\right)+\sum_{x \in W} \operatorname{sgn}\left(\operatorname{det} \tilde{f}^{\prime}(x)\right), \\
\operatorname{deg}\left(\tilde{F}_{1}\right)=\operatorname{deg}\left(\tilde{f}_{0}\right)+\sum_{x \in V} \operatorname{sgn}\left(\operatorname{det} \tilde{f}^{\prime}(x)\right) \text { and } \\
\operatorname{deg}\left(\tilde{F}_{2}\right)=\operatorname{deg}\left(\tilde{f}_{0}\right)+\sum_{x \in W} \operatorname{sgn}\left(\operatorname{det} \tilde{f}^{\prime}(x)\right) .
\end{gathered}
$$

The result follows from (3.7), (3.8) and (3.9) and the homotopy invariance of the degree.

Theorem 12. Let $f:\left(S^{n}, S^{n-1}\right) \rightarrow\left(S^{n}, S^{n-1}\right)$ be a continuous map. If $F_{1}$ and $F_{2}$ are continuous extensions of $f_{+}$and $f_{-}$respectively, satisfying (3.1), then (3.2) holds.

Proof. As a consequence of Lemma 11 it will be sufficient to find a smooth map $\tilde{f}:\left(S^{n}, S^{n-1}\right) \rightarrow$ $\left(S^{n}, S^{n-1}\right)$ homotopic to $f$ as maps of pairs, and such that the corresponding maps $\tilde{f}_{+}$and $\tilde{f}_{-}$ have smooth extensions $\tilde{F}_{1}$ and $\tilde{F}_{2}$, homotopic $F_{1}$ and $F_{2}$ and satisfying the assumption (3.1) of Lemma 11.

Let $\tilde{f}_{0}: S^{n-1} \rightarrow S^{n-1}$ be a smooth map homotopic to $f_{0}$. By Lemma 10 there exists a smooth map $g:\left(S_{+}^{n}, S^{n-1}\right) \rightarrow\left(S^{n}, S^{n-1}\right)$ homotopic to $f_{+}:\left(S_{+}^{n}, S^{n-1}\right) \rightarrow\left(S^{n}, S^{n-1}\right)$ as maps of pairs. Hence, $\tilde{f}_{0}$ and $g_{0}$ are homotopic and, since they are smooth, there exists a smooth homotopy $H_{t}: S^{n-1} \rightarrow S^{n-1}$ such that $H_{0}=\tilde{f}_{0}$ and $H_{1}=g_{0}$. 
We extend $\tilde{f}_{0}$ to a map $\tilde{f}_{+}: S_{+}^{n} \rightarrow S^{n}$ letting

$$
\tilde{f}_{+}(x)=\left\{\begin{array}{lll}
\pi\left(\tilde{f}_{0}(p), t\right) & \text { if } & x=\pi(p, t) \in \pi\left(S_{[0,1 / 3]}\right) \\
\pi\left(H_{6 t-2}(p), t\right) & \text { if } \quad x=\pi(p, t) \in \pi\left(S_{(1 / 3,1 / 2]}\right) \\
\tilde{g}(x) & \text { if } \quad x \in \pi\left(S_{(1 / 2,1]}\right),
\end{array}\right.
$$

where $\tilde{g}$ is obtained by rescaling $g$ in the obvious way.

In a symmetric way, it is possible to extend $\tilde{f}_{+}$to $S_{-}^{n}$ ending up with a smooth function $\tilde{f}: S^{n} \rightarrow S^{n}$ homotopic to $f$.

To construct a smooth extension $\tilde{F}_{1}$ of $\tilde{f}_{+}$such that (3.1) holds we define $\tilde{F}_{1}: \pi\left(S_{[-1 / 2,1]}\right) \rightarrow$ $S^{n}$ as follows

$$
\tilde{F}_{1}=\left\{\begin{array}{lll}
\tilde{f}_{+}(x) & \text { if } \quad x \in S_{+}^{n} \\
\pi\left(\tilde{f}_{0}(p), t\right) & \text { if } \quad x=\pi(p, t) \in \pi\left(S_{[-1 / 2,0)}\right) .
\end{array}\right.
$$

By proceeding in an analogous way as in the construction of $\tilde{f}$ it is possible to extend $\tilde{F}_{1}$ to a smooth map $\tilde{F}_{1}: S^{n} \rightarrow S^{n}$, homotopic to $F_{1}$ and such that

$$
\tilde{F}_{1}\left(\pi\left(S_{[-1,-1 / 2)}\right)\right) \subset \pi\left(S_{[-1,-1 / 2)}\right) .
$$

The construction $\tilde{F}_{2}$ is analogous.

The next result is a nice consequence of Theorem 12 .

Proposition 13. Assume that $f: S^{n} \rightarrow S^{n}$ is a continuous map such that $f=J \circ f \circ J$, where $J: S^{n} \rightarrow S^{n}$ is given by $J(\pi(p, t))=\pi(p,-t)$. Then $f\left(S^{n-1}\right) \subset S^{n-1}$ and

$$
\operatorname{deg}(f) \equiv \operatorname{deg}\left(f_{0}\right) \quad(\bmod 2) .
$$

Proof. We define the extension $F_{1}: S^{n} \rightarrow S^{n}$ of $f_{+}$by

$$
F_{1}(x)=\left\{\begin{array}{lll}
f_{+}(x) & \text { if } & x \in S_{+}^{n} \\
\pi\left(f_{0}(p), t\right) & \text { if } & x=\pi(p, t) \in \pi\left(S_{(0,-1)}\right) \\
p_{S} & \text { if } & x=p_{S},
\end{array}\right.
$$

where $p_{S}$ denotes the south pole of $S^{n}$.

The extension $F_{2}$ of $f_{-}$is defined in an analogous way. On the other hand, since $f=J \circ f \circ J$ it follows that $\operatorname{deg}\left(F_{1}\right)=\operatorname{deg}\left(F_{2}\right)$ and, as a consequence, Theorem 12 ensures that

$$
\operatorname{deg}(f)+\operatorname{deg}\left(f_{0}\right)=2 \operatorname{deg}\left(F_{1}\right)
$$

\section{Mayer-Vietoris index formula for Euclidean SPaces}

In this section we prove the main results of this note. In particular we will see that if $f:\left(X ; X_{1}, X_{2}\right) \rightarrow\left(X ; X_{1}, X_{2}\right)$ is a continuous map with compact fixed point set and either $\left(X ; X_{1}, X_{2}\right)$ is $\left(E^{n} ;, E_{+}^{n}, E_{-}^{n}\right)$ or admits a good Euclidean embedding, then (0.1) holds.

Let $f:\left(E^{n} ; E_{+}^{n}, E_{-}^{n}\right) \rightarrow\left(E^{n} ; E_{+}^{n}, E_{-}^{n}\right)$ be a continuous map. We will use the notation

$$
f_{+}:=\left.f\right|_{E_{+}^{n}}: E_{+}^{n} \rightarrow E_{+}^{n}, f_{-}:=\left.f\right|_{E_{-}^{n}}: E_{-}^{n} \rightarrow E_{-}^{n} \text { and } f_{0}:=\left.f\right|_{E_{0}^{n}}: E_{0}^{n} \rightarrow E_{0}^{n} .
$$

Theorem 14. Suppose $f:\left(E^{n} ; E_{+}^{n}, E_{-}^{n}\right) \rightarrow\left(E^{n} ; E_{+}^{n}, E_{-}^{n}\right)$ is a continuous map satisfying that $\operatorname{Fix}(f)$ is compact. Then (0.1) holds.

Proof. The case $n=1$ is easier and is left to the reader, so we will assume that $n \geq 2$. Since Fix $(f)$ is compact, there exists a metric closed ball $B$ in $E^{n}$ centered at the origin containing $\operatorname{Fix}(f)$ in its interior. Consider

$$
r_{1}: E^{n} \rightarrow E_{+}^{n} \quad \text { and } \quad r_{2}: E^{n} \rightarrow E_{-}^{n},
$$

be the natural retractions and

$$
j_{1}: E_{+}^{n} \hookrightarrow E^{n} \quad \text { and } \quad j_{2}: E_{-}^{n} \hookrightarrow E^{n},
$$

the inclusions. We consider the maps $F_{i}: E^{n} \rightarrow E^{n}, i=1,2$ given by

$$
F_{1}:=j_{1} \circ f_{+} \circ r_{1}, \quad F_{2}:=j_{2} \circ f_{-} \circ r_{2} .
$$

The index of the maps $f, F_{1}, F_{2}$ and $f_{0}$ agree with the degree of the maps $\Phi, \Phi_{i}: \partial B \rightarrow S^{n-1}$, $i=1,2$ and $\Phi_{0}: \partial B \cap E_{0}^{n} \rightarrow S^{n-2}$ given by

$$
\Phi(x)=\frac{x-f(x)}{\|x-f(x)\|}, \quad \Phi_{i}(x)=\frac{x-F_{i}(x)}{\left\|x-F_{i}(x)\right\|}, \quad \text { and } \quad \Phi_{0}(x)=\frac{x-f_{0}(x)}{\left\|x-f_{0}(x)\right\|} .
$$


Notice that, if we denote by $\partial B_{+}$and $\partial B_{-}$the intersections of $\partial B$ with $E_{+}^{n}$ and $E_{-}^{n}$ respectively, then $\left.\Phi_{1}\right|_{\partial B_{+}}=\left.\Phi\right|_{\partial B_{+}},\left.\Phi_{2}\right|_{\partial B_{-}}=\left.\Phi\right|_{\partial B_{-}}$and $\Phi_{0}=\left.\Phi\right|_{\partial B \cap E_{0}^{n}}$.

On the other hand, it is clear that the natural radial diffeomorphism from $S^{n-1}$ onto $\partial B$ is a diffeomorphism of triplets $h:\left(S^{n-1} ; S_{+}^{n-1}, S_{-}^{n-1}\right) \rightarrow\left(\partial B ; \partial B_{+}, \partial B_{-}\right)$. It is straightforward to see that the maps $\Phi \circ h$ and $\Phi_{i} \circ h, i=1,2$, satisfy the assumptions of Theorem 12 . The result follows by combining these remarks with the fact that the degree of a composition is the product of the degrees and that $\operatorname{deg}(h)=\operatorname{deg}\left(\left.h\right|_{S^{n-2}}\right)=1$.

Remark 15. Notice that, from the proof of Theorem 14, it follows that if $f:\left(E^{n} ; E_{+}^{n}, E_{-}^{n}\right) \rightarrow$ $\left(E^{n} ; E_{+}^{n}, E_{+}^{n}\right)$ is a continuous map and $U$ is a metric open ball with no fixed points on its boundary, then

$$
i(f, U)+i\left(f_{0}, U \cap E_{0}^{n}\right)=i\left(f_{+}, U \cap E_{+}^{n}\right)+i\left(f_{-}, U \cap E_{-}^{n}\right) .
$$

An inmediate consequence of this fact is that, if 0 is an isolated fixed point of $f$ then

$$
i(f, 0)+i\left(f_{0}, 0\right)=i\left(f_{+}, 0\right)+i\left(f_{-}, 0\right) .
$$

Corollary 16. Assume that $f:\left(E^{n} ; E_{+}^{n}, E_{-}^{n}\right) \rightarrow\left(E^{n} ; E_{+}^{n}, E_{-}^{n}\right)$ is smooth and 0 is an isolated fixed point. If $f_{0}^{\prime}(0)-I: E_{0}^{n} \rightarrow E_{0}^{n}$ is a non-singular linear transformation then $i(f, 0) \in$ $\{-1,0,1\}$.

Proof. It follows from [5, Theorem 5.1] that $i\left(f_{ \pm}, 0\right) \in\left\{0, i\left(f_{0}, 0\right)\right\}$. Then, it follows from Remark 15 that $i(f, 0) \in\left\{0, \pm i\left(f_{0}, 0\right)\right\}$. The result follows since $i\left(f_{0}, 0\right)= \pm 1$.

The remaining part of the section deals with the concept of good Euclidean embedding.

Definition 17. We say that a triplet $\left(X ; X_{1}, X_{2}\right)$ admits a good Euclidean embedding if:

(1) there exists $n \geq 1$ and an embedding $e: X \rightarrow E^{n}$ such that $e\left(X_{1}\right) \subset E_{+}^{n}$ and $e\left(X_{2}\right) \subset E_{-}^{n}$,

(2) there exists a retraction $r: E^{n} \rightarrow e(X)$ such that

$$
r\left(E_{+}^{n}\right)=e\left(X_{1}\right), \quad \text { and } \quad r\left(E_{-}^{n}\right)=e\left(X_{2}\right) .
$$

Remark 18. If the triplet $\left(X ; X_{1}, X_{2}\right)$ admits a good Euclidean embedding, it is straightforward from Definition 17 that $\left.r\right|_{E_{0}^{n}}$ is also a retraction onto $X_{0}$. As a consequence, $X$ and $X_{i}, i=0,1,2$ are ENR's being homeomorphic to retracts of ENR's.
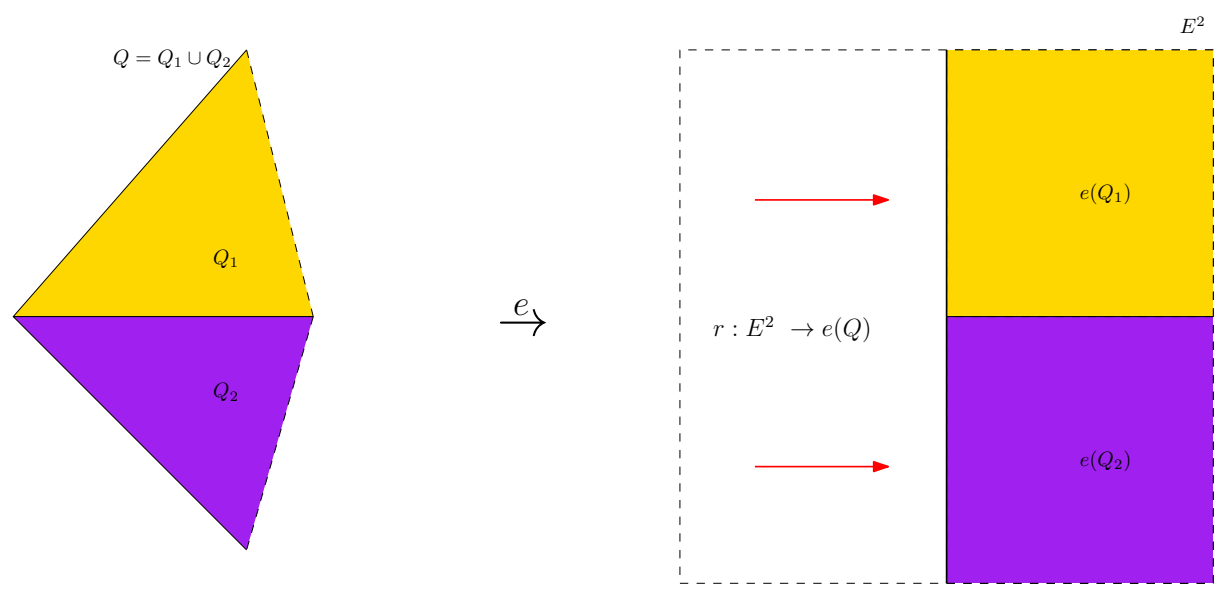

Figure 1. Example of a good Euclidean embedding of a triplet $\left(Q ; Q_{1}, Q_{2}\right)$ in $E^{2}$

Theorem 19. Let $f:\left(X ; X_{1}, X_{2}\right) \rightarrow\left(X ; X_{1}, X_{2}\right)$ be a continuous map satisfying that $\operatorname{Fix}(f)$ is compact and suppose that the triplet $\left(X ; X_{1}, X_{2}\right)$ admits a good Euclidean embedding. Then (0.1) holds.

Proof. Since it should not cause any confusion we will identify $X$ and $X_{i}, i=0,1,2$ with $e(X)$ and $e\left(X_{i}\right), i=0,1,2$ respectively.

Let $e_{0}: X_{0} \rightarrow E_{0}^{n}, e_{1}: X_{1} \rightarrow E_{+}^{n}, e_{2}: X_{2} \rightarrow E_{-}^{n}$ the respective restrictions of the embedding $e$ to $X_{i}, i=0,1,2$ and $k_{1}: E_{+}^{n} \hookrightarrow E^{n}, k_{2}: E_{-}^{n} \hookrightarrow \bar{E}^{n}$ the inclusions. We define

$$
F=e \circ f \circ r: E^{n} \rightarrow E^{n} \text {. }
$$


Notice that $\operatorname{Fix}(F)=\operatorname{Fix}(f)$ and, as a consequence, it is compact. It follows from Theorem 14 that

$$
i\left(F, E^{n}\right)+i\left(F_{0}, E_{0}^{n}\right)=i\left(F_{+}, E_{+}^{n}\right)+i\left(F_{-}, E_{-}^{n}\right) .
$$

On the other hand, from the definition of the fixed point index we have $i\left(F, E^{n}\right)=i(F, X)$ and, since

$$
F_{0}=\left.e_{0} \circ f_{0} \circ r\right|_{E_{0}^{n}}
$$

and $E_{0}^{n}=E^{n-1} \times\{0\}$, it follows that

$$
i\left(F_{0}, E_{0}^{n}\right)=i\left(f_{0}, X_{0}\right) .
$$

The remaining part of the proof is to see that $i\left(F_{+}, E_{+}^{n}\right)=i\left(f_{1}, X_{1}\right)$ and $i\left(F_{-}, E_{-}^{n}\right)=i\left(f_{2}, X_{2}\right)$.

Let $r_{1}: E^{n} \rightarrow E_{+}^{n}$ be the natural retraction. From the definition of the fixed point index we have

$$
i\left(F_{+}, E_{+}^{n}\right)=i\left(k_{1} \circ F_{+} \circ r_{1}, E^{n}\right)=i\left(k_{1} \circ\left(\left.e_{1} \circ f_{+} \circ r\right|_{E_{+}^{n}}\right) \circ r_{1}, E^{n}\right),
$$

Since $\left.r\right|_{E_{+}^{n}} \circ r_{1}: E^{n} \rightarrow X_{1}$ is a retraction and $k_{1} \circ e_{1}: X_{1} \rightarrow E^{n}$ is an embedding, it follows that $i\left(F_{+}, E_{+}^{n}\right)=i\left(f_{1}, X_{1}\right)$. The equality $i\left(F_{-}, E_{-}^{n}\right)=i\left(f_{1}, X_{1}\right)$ is proven in an analogous way.

5. Sectorial decomposition - generalization of the Poincaré-Bendixson index formula

In this section we generalize the Poincaré-Bendixson index formula for vector fields to the context of continuous maps $f: E^{2} \rightarrow E^{2}$ whose fixed point set is compact. In addition we present some applications of this formula and the previous results to orientation preserving homeomorphisms of $E_{+}^{2}$ satisfying that $\operatorname{Fix}(f)=\{0\}$.

We will say that $Q \subset E^{2}$ is a sector if it is a region bounded by two half-lines with common starting point (including the half-lines).

Remark 20. Notice that the union of two sectors whose intersection is one their boundary half-lines is also a sector.

Definition 21. A continuous map $f: E^{2} \rightarrow E^{2}$ has a sectorial decomposition $\left\{Q_{1}, \ldots, Q_{p}\right\}$, $p \geq 2$, if there exist different points $z_{1}, \ldots, z_{p} \in S^{1}$, counter clockwise ordered on the circle such that for each $i=1, \ldots, p-1, Q_{i}$ is the sector bounded by the half-lines $L_{i}$ and $L_{i+1}$ starting at the origin and passing through $z_{i}$ and $z_{i+1}$ respectively, $Q_{p}$ is the sector bounded by the half-lines $z_{p}$ and $z_{1}$ and $f\left(Q_{i}\right) \subset Q_{i}$ for each $i=1, \ldots, p$.

Remark 22. From Definition 21 it readily follows that $f\left(L_{i}\right) \subset L_{i}$ for all $i=1, \ldots, p$.

Lemma 23. Let $f: E^{2} \rightarrow E^{2}$ be a continuous map satisfying that $\operatorname{Fix}(f)$ is compact. If $f$ has a sectorial decomposition $\left\{Q_{1}, \ldots, Q_{p}\right\}$, then

$$
i\left(f, E^{2}\right)=1+\sum_{i=1}^{p} i\left(\left.f\right|_{Q_{i}}, Q_{i}\right)-\sum_{i=1}^{p} i\left(\left.f\right|_{L_{i}}, L_{i}\right)
$$

Proof. It is easy to check that in general, if $Q_{1}$ and $Q_{2}$ are two sectors whose intersection is one of their boundary half-lines $L$, the triplet $\left(Q_{1} \cup Q_{2} ; Q_{1}, Q_{2}\right)$ admits a good Euclidean embedding (see figure 1). Then, from Theorem 19 it follows that

$$
i\left(\left.f\right|_{Q_{1} \cup Q_{2}}, Q_{1} \cup Q_{2}\right)+i\left(\left.f\right|_{L}, L\right)=i\left(\left.f\right|_{Q_{1}}, Q_{1}\right)+i\left(\left.f\right|_{Q_{2}}, Q_{2}\right) .
$$

Now, suppose that $f$ has sectorial decomposition $\left\{Q_{1}, \ldots, Q_{p}\right\}$. Then, if we consider $Q=$ $Q_{1} \cup \ldots \cup Q_{p-1}$, Remark 20 and (5.1) together with and induction argument ensure that

$$
i\left(\left.f\right|_{Q}, Q\right)=-\sum_{i=2}^{p-1} i\left(\left.f\right|_{L_{i}}, L_{i}\right)+\sum_{i=1}^{p-1} i\left(\left.f\right|_{Q_{i}}, Q_{i}\right) .
$$

On the other hand, again from Theorem 19 we get

$$
i\left(f, E^{2}\right)+i\left(\left.f\right|_{L_{1} \cup L_{p}}, L_{1} \cup L_{p}\right)=i\left(\left.f\right|_{Q}, Q\right)+i\left(\left.f\right|_{Q_{p}}, Q_{p}\right),
$$

and

$$
i\left(\left.f\right|_{L_{1} \cup L_{p}}, L_{1} \cup L_{p}\right)+1=i\left(\left.f\right|_{L_{1}}, L_{1}\right)+i\left(\left.f\right|_{L_{p}}, L_{p}\right) .
$$

Hence, the result follows from (5.2), (5.3) and (5.4).

Remark 24. Assume that a continuous map $f: E^{2} \rightarrow E^{2}$ admits a sectorial decomposition $\left\{Q_{1}, \ldots, Q_{p}\right\}$ and $\operatorname{Fix}(f)=\{0\}$. Since for each $i=1, \ldots, p, L_{i}$ may be embedded in $\mathbb{R}$ as $[0,+\infty)$ an easy computation shows that

$$
i\left(\left.f\right|_{L_{i}}, 0\right) \in\{0,1\} .
$$


Corollary 25. Assume that $f: E^{2} \rightarrow E^{2}$ admits a sectorial decomposition $\left\{Q_{1}, \ldots, Q_{p}\right\}$ and $\operatorname{Fix}(f)=\{0\}$. If we denote by $l=\operatorname{card}\left\{i \in\{1, \ldots, p\}: i\left(\left.f\right|_{L_{i}}, 0\right)=1\right\}$, then

$$
i(f, 0)=1-l+\sum_{i=1}^{p} i\left(\left.f\right|_{Q_{i}}, 0\right) .
$$

If, in addition $i\left(\left.f\right|_{Q_{i}}, 0\right) \in\{0,1\}$ for each $i=1, \ldots, p$ and $e=\operatorname{card}\left\{i \in\{1, \ldots, p\}: i\left(\left.f\right|_{Q_{i}}, 0\right)=\right.$ $1\}$, then

$$
i(f, 0)=1-l+e .
$$

Remark 26. One can think about (5.5) as a kind of Euler characteristic formula. In the classical Poincaré-Bendixson index formula (see [10, Proposition 6.32, pp. 179] and [17]) the number of sectors would be $p=2 n$ and

$$
i\left(\left.f\right|_{L_{i}}, 0\right)= \begin{cases}1 & \text { if } i \text { is even } \\ 0 & \text { if } i \text { is odd }\end{cases}
$$

hence $l=n$. The sectors with index 1 are called elliptic sectors and the sectors with index 0 are called hyperbolic sectors. Hence, the number of hyperbolic sectors is $h=2 n-e$. It follows that $l=\frac{e+h}{2}$, so

$$
i(f, 0)=1-\frac{e+h}{2}+e=1+\frac{e-h}{2} .
$$

Let $U \subset E^{2}$ be an open set. A homeomorphism $f: U \rightarrow U$ is called free provided whenever $D \subset U$ is a topological disk such that $f(D) \cap D=\emptyset$, then $f^{i}(D) \cap f^{j}(D)=\emptyset$ for all $i \neq j$. Obviously, the only periodic points of a free homeomorphism are fixed points.

Proposition 27. Let $f: E_{+}^{2} \rightarrow E_{+}^{2}$ be an orientation preserving homeomorphism such that $\operatorname{Fix}(f)=\{0\}$. Then $\left.f\right|_{E_{+}^{2} \backslash E_{0}^{2}}$ is a free homeomorphism, and, hence, $f$ has no other periodic points than the fixed point 0 and

$$
i\left(f^{n}, 0\right)=i(f, 0), \quad n>0 .
$$

Proof. We extend $f=\left(f_{1}, f_{2}\right)$ to the homeomorphism $F: E^{2} \rightarrow E^{2}$ given by

$$
F(x, y)=\left\{\begin{array}{lll}
f(x, y) & \text { if } & (x, y) \in E_{+}^{2} \\
\left(f_{1}(x,-y),-f_{2}(x,-y)\right) & \text { if } & (x, y) \in E_{-}^{2} .
\end{array}\right.
$$

From Theorem 14 combined with the fact that $i\left(F_{+}, 0\right)=i\left(F_{-}, 0\right)=i(f, 0)$ it follows

$$
i(F, 0)+i\left(f_{0}, 0\right)=2 i(f, 0)
$$

Case 1. Assume that $i\left(f_{0}, 0\right)=0$. Then, from (5.6) it follows

$$
i(F, 0)=2 i(f, 0),
$$

so $i(F, 0)$ is even. Since $F$ is an orientation preserving homeomorphism with the unique fixed point at the origin and $i(F, 0) \neq 1$ it follows from Theorem 3 in [4] that $F$ is a free homeomorhism and $i\left(F^{n}, 0\right)=i(F, 0)$ for $n>0$, so the result follows.

Case 2. Either $\left(i\left(f_{0}, 0\right)=1\right.$ and $\left.i(f, 0) \neq 1\right)$ or $\left(i\left(f_{0}, 0\right)=-1\right.$ and $\left.i(f, 0) \neq 0\right)$. It follows from (5.6) that $i(F, 0) \neq 1$, so we can use the same arguments as in the proof of Case 1.

Case 3. $i\left(f_{0}, 0\right)=1$ and $i(f, 0)=1$. We extend $f$ to a homeomorphism $H: E^{2} \rightarrow E^{2}$ such that $\operatorname{Fix}\left(H^{n}\right)=\{0\}$ for $n>0$ and $H$ admits a sectorial decomposition $\left\{Q_{1}, Q_{2}, Q_{3}\right\}$ where

$$
Q_{1}=E_{+}^{2}, \quad Q_{2}=\left\{(x, y) \in E^{2}: x \leq 0, y \leq 0\right\}, \quad Q_{3}=\left\{(x, y) \in E^{2}: x \geq 0, y \leq 0\right\}
$$

with rays

$$
L_{1}=\{(x, 0): x \geq 0\}, \quad L_{2}=\{(x, 0): x \leq 0\}, \quad L_{3}=\{(0, y): y \leq 0\},
$$

and such that $i\left(\left(\left.H\right|_{L_{3}}\right)^{n}, 0\right)=0(n>0)$. Observe that such an $H$ must satisfy $i\left(\left(\left.H\right|_{L_{i}}\right)^{n}, 0\right)=1$ for $i=1,2$ and $n>0$, since $i\left(f_{0}, 0\right)=1$. To define $H$, consider $f_{1}$ to be the first component of $f$ and let $h:(-\infty, 0] \rightarrow(-\infty, 0]$ be a homeomorphism such that $\lim _{n \rightarrow+\infty} h^{n}(x)=-\infty$ for $x \neq 0$. We define

$$
H(x, y)= \begin{cases}f(x, y) & \text { if }(x, y) \in E_{+}^{2} \\ \left(f_{1}(x, 0), h(y)\right) & \text { if }(x, y) \in E_{-}^{2} .\end{cases}
$$

Since $\left.H\right|_{Q_{i}}(i=2,3)$ are in the Case 1 , then $i\left(\left(\left.H\right|_{Q_{i}}\right)^{n}, 0\right)=i\left(\left.H\right|_{Q_{i}}, 0\right)$ for $n>0$ and $i=2,3$. It follows from Lemma 23 that

$$
i(H, 0)=1+i\left(H_{+}, 0\right)+i\left(\left.H\right|_{Q_{2}}, 0\right)+i\left(\left.H\right|_{Q_{3}}, 0\right)-2=2 i\left(\left.H\right|_{Q_{2}}, 0\right) \neq 1,
$$


since $i\left(\left.H\right|_{E_{+}^{2}}, 0\right)=1$ and $i\left(\left.H\right|_{Q_{2}}, 0\right)=i\left(\left.H\right|_{Q_{3}}, 0\right)$. We can use the arguments like in the proof of Case 1.

Case 4. If $i\left(f_{0}, 0\right)=-1$ and $i(f, 0)=0$ we are in a dual situation to the Case 3 .

Corollary 28. Assume that $f: E^{2} \rightarrow E^{2}$ is an orientation preserving homeomorphism of the plane with the origin as its uniqe fixed point. If $f$ has an invariant sector, then the sequence $\left\{i\left(f^{n}, 0\right)\right\}_{n \geq 1}$ is well-defined and constant.

If we consider orientation and area preserving homeomorphisms $f: E_{+}^{2} \rightarrow E_{+}^{2}$ which have the origin as their unique fixed point, we can obtain upper bounds for $i(f, 0)$ by combining Theorem 14 with some results of [25].

Proposition 29. Let $f: E_{+}^{2} \rightarrow E_{+}^{2}$ be an orientation and area preserving homeomorphism and $\operatorname{Fix}(f)=\{0\}$. Then

(1) if $i\left(f_{0}, 0\right) \in\{0,-1\}$ then $i(f, 0) \leq 0$,

(2) if $i\left(f_{0}, 0\right)=1$ then $i(f, 0) \leq 1$.

Proof. Let $F: E^{2} \rightarrow E^{2}$ be a homeomorphism defined in the proof of Proposition 27. Since $F$ is area preserving it follows from [25] that $i(F, 0) \leq 1$. Hence the result follows using this fact in (5.6).

6. IsOlated INVARIANT SETS IN THE INVARIANT SUbSPaCe.

The aim of this section is to give an application of Theorem 14 to the index of a continuous map $f:\left(E^{n} ; E_{+}^{n}, E_{-}^{n}\right) \rightarrow\left(E^{n} ; E_{+}^{n}, E_{-}^{n}\right)$ in an isolated invariant set. For this purpose we start by recalling some definitions about these sets.

Let $(X, d)$ be a locally compact metric space and $f: X \rightarrow X$ a continuous map.

The sequence $\sigma: \mathbb{Z}_{-} \rightarrow X$ is said to be a left solution to $f$ through $x$ if $\sigma(0)=x$ and $f(\sigma(i-1))=\sigma(i)$ for each $i \in \mathbb{Z}_{-}$.

Given $N \subset X$, by $\operatorname{Inv}_{f}^{+} N, \operatorname{Inv}_{f}^{-} N$, and $\operatorname{Inv}_{f} N$ we shall denote, respectively, the positively invariant, negatively invariant and invariant part of $N$ relative to $f$, defined by

$$
\begin{gathered}
\operatorname{Inv}_{f}^{+} N=\left\{x \in X: f^{i}(x) \in X \text { for all } i \in \mathbb{Z}_{+}\right\}, \\
\operatorname{Inv}_{f}^{-} N=\left\{x \in X: \exists \sigma: \mathbb{Z}_{-} \rightarrow N \text { left solution to } f \text { through } x\right\}, \\
\operatorname{Inv}_{f} N=\operatorname{Inv}_{f}^{+} N \cap \operatorname{Inv}_{f}^{-} N .
\end{gathered}
$$

A set $S$ is said to be invariant if $f(S)=S$. This is easily seen to be equivalent to $S=\operatorname{Inv}_{f} S$. We say that $S$ is an isolated invariant set if it admits a compact neighborhood $N$ such that $S=\operatorname{Inv}_{f} N$. Such a neighborhood $N$ is said to be an isolating neighborhood of $S$. If $X$ is an ENR then $i(f, S)$ is defined as the fixed point index of $f$ on the interior of any isolating neighborhood of $S$.

We define the stable and unstable sets of the compact invariant set $S$ by

$$
W^{s}(S, f)=\left\{x \in X: \lim _{i \rightarrow \infty} d\left(f^{i}(x), S\right)=0\right\}
$$

$W^{u}(S, f)=\left\{x \in X: \exists \sigma\right.$ left solution of $f$ through $x$ such that $\left.\lim _{i \rightarrow \infty} d(\sigma(-i), S)=0\right\}$.

Proposition 30. Let $f:\left(E^{n} ; E_{+}^{n}, E_{-}^{n}\right) \rightarrow\left(E^{n} ; E_{+}^{n}, E_{-}^{n}\right)$ be a contiuous map and $S \subset E_{0}^{n}$ an isolated invariant set for $f$ such that $\operatorname{Fix}(f) \subset S$.

(1) If $W^{u}(S, f) \subset E_{+}^{n}$ then $i(f, S)=i\left(f_{+}, S\right)$. Moreover, if $W^{u}(S, f) \subset E_{0}^{n}$ then

$$
i(f, S)=i\left(f_{+}, S\right)=i\left(f_{-}, S\right)=i\left(f_{0}, S\right) .
$$

(2) If $W^{s}(S, f) \subset E_{-}^{n}$ then $i\left(f_{+}, S\right)=0$. Moreover, if $W^{s}(S, f) \subset E_{0}^{n}$ then

$$
i(f, S)=-i\left(f_{0}, S\right), \quad i\left(f_{ \pm}, S\right)=0 .
$$

(3) If $W^{s}(S, f) \subset E_{-}^{n}$ and $W^{u}(S, f) \subset E_{+}^{n}$ then $i(f, S)=0$.

Proof. Suppose that $W^{u}(S, f) \subset E_{+}^{n}$. As a consequence, $W^{u}\left(S, f_{-}\right) \subset E_{0}^{n}$ and it follows from $[31$, Corollary 6] that

$$
i\left(f_{-}, S\right)=i\left(f_{0}, S\right) .
$$

Thus, the first part of (1) follows from this together with Theorem 14. If additionally, $W^{u}(S, f) \subset$ $E_{0}^{n}$ then $i\left(f_{-}, S\right)=i(f, S)$ by symmetry and, hence, (1) holds. 
If $W^{s}(S, f) \subset E_{-}^{n}$ then $W^{s}\left(S, f_{+}\right) \subset E_{0}^{n}$ and, hence, [31, Corollary 6] ensures that

$$
i\left(f_{+}, S\right)=0 \text {. }
$$

In order to finish the proof of (2), suppose that $W^{s}(S, f) \subset E_{0}^{n}$. Reasoning in an analogous way we get that

$$
i(f, S)=-i\left(f_{0}, S\right), \quad i\left(f_{ \pm}, S\right)=0 .
$$

One can check that (3) follows by combining (1) and (2).

Remark 31. Let $f:\left(E^{3} ; E_{+}^{3}, E_{-}^{3}\right) \rightarrow\left(E^{3}, E_{+}^{3}, E_{-}^{3}\right)$ be a continuous map with the origin being an isolated invariant set. Proposition 30 together with $[22,28]$ sheds some light on the behaviour of the sequence $\left(i\left(f^{n}, 0\right)\right)_{n \geq 1}$ in dimension 3. Suppose that $W^{u}\left(0, f^{n}\right) \subset E_{0}^{3}$ for every $n \geq 1$. Hence, item (1) in Proposition 30 ensures that $i\left(f^{n}, 0\right)=i\left(f_{0}^{n}, 0\right)$ for each $n \geq 1$.

Following [14] we say that the isolated invariant set $\{0\}$ is a sink provided there exists an isolating neighborhood $N$ of 0 such that $f(N) \subset N$. On the other hand, $\{0\}$ is called a source if there exists an isolating neighborhood $N$ such that $f(\partial N) \cap \operatorname{int}(N)=\emptyset$ and the forward orbit of every point $x \in N \backslash\{0\}$ eventually exits $N$.

If $\{0\}$ is neither a sink nor a source with respect to $f_{0}$ and $f_{0}$ is an orientation preserving homeomorphism, then there exist positive integers $r, q$, such that for each $n \geq 1$ (compare [22])

$$
i\left(f^{n}, 0\right)=i\left(f_{0}^{n}, 0\right)= \begin{cases}1-r q & \text { if } q \mid n \\ 1 & \text { otherwise. }\end{cases}
$$

If $f_{0}$ is an orientation reversing homeomorphism instead of orientation preserving one can use [28] to get that there are integers $\delta \in\{0,1,2\}$ and $q$ such that

$$
i\left(f^{n}, 0\right)=i\left(f_{0}^{n}, 0\right)= \begin{cases}1-\delta & \text { if } n \text { is odd } \\ 1-\delta-2 q & \text { if } n \text { is even. }\end{cases}
$$

If the origin 0 is a source for $f_{0}$ then by [14] there exists an integer $d$ such that $i\left(f^{n}, 0\right)=$ $i\left(f_{0}^{n}, 0\right)=d^{n}$ for any $n \geq 1$.

\section{Maps of $E^{3}$ Symmetric with Respect $E_{0}^{3}$}

In this section we consider continuous maps $f:\left(E^{3} ; E_{+}^{3}, E_{-}^{3}\right) \rightarrow\left(E^{3} ; E_{+}^{3}, E_{-}^{3}\right)$ which are symmetric with respect to $E_{0}^{3}$, i.e., $f=J \circ f \circ J$, where $J(x, y, z)=(x, y,-z)$.

The following result deals with the fixed point index of isolated fixed points. Its first part is an application of Theorem 14 to the index of an isolated stable fixed point. The index of stable fixed points has been extensively studied. For instance, Bonatti and Villadelprat (see [2]) have shown that, in dimension greather or equal to 3, the index of a stable critical point of a vector field can be any integer. Dancer and Ortega [7] proved that for any orientation preserving homeomorphism of the plane such that 0 is an isolated and stable fixed point, the fixed point index at 0 is equal to 1. The same was proved in [27] for orientation reversing homeomorphisms. Let us mention that if 0 is a sink, its index is equal to 1 in any dimension.

Proposition 32. Let $f:\left(E^{3} ; E_{+}^{3}, E_{-}^{3}\right) \rightarrow\left(E^{3} ; E_{+}^{3}, E_{-}^{3}\right)$ be a continuous map symmetric with respect to $E_{0}^{3}$ satisfying that the origin is an isolated fixed point. Then

(1) If $f_{0}$ is a homeomorphism and 0 is stable with respect to $f_{0}$ then $i(f, 0)$ is odd.

(2) If 0 is an isolated fixed point of $f^{n}$ for all $n \geq 1$ and $f_{0}$ is orientation preserving homeomorphism then there is an integer $p \in \mathbb{Z} \backslash\{0,2\}$ such that

$$
i\left(f^{n}, 0\right) \in \begin{cases}i\left(f_{0}, 0\right)+2 \mathbb{Z} & \text { if } i\left(f_{0}, 0\right) \neq 1 \\ 1+2 \mathbb{Z} & \text { if } i\left(f_{0}^{n}, 0\right)=1, \\ p+2 \mathbb{Z} & \text { if } i\left(f_{0}^{n}, 0\right)=p .\end{cases}
$$

(3) If 0 is an isolated fixed point of $f^{n}$ for all $n \geq 1$ and $f_{0}$ is orientation reversing homeomorphism then for every odd $n \geq 1$

$$
i\left(f^{n}, 0\right) \in \begin{cases}2 \mathbb{Z} & \text { if } i\left(f_{0}, 0\right)=0 \\ 1+2 \mathbb{Z} & \text { if } i\left(f_{0}, 0\right) \neq 0 .\end{cases}
$$

Proof. Observe that if 0 is an isolated fixed point of $f^{n}$ for some $n \geq 1$ then it follows from the proof of Theorem 14 that

$$
i\left(f^{n}, 0\right)+i\left(f_{0}^{n}, 0\right)=2 i\left(f_{+}^{n}, 0\right)
$$

and, as a consequence, $i\left(f^{n}, 0\right) \equiv i\left(f_{0}^{n}, 0\right)(\bmod 2)$. The case $(1)$ follows since $i\left(f_{0}, 0\right)=1$ by $[7,27]$. 
We prove (2) and (3). Assume that 0 is an isolated fixed point of $f^{n}$ for every $n \geq 1$ and let $f_{0}$ be orientation preserving. If $i(f, 0) \neq 1$ then $i\left(f_{0}, 0\right)$ is also different from 1 and, hence, $i\left(f_{0}^{n}, 0\right)=i\left(f_{0}, 0\right)$ for every $n \geq 1$ by [4]. As a consequence, $i\left(f^{n}, 0\right) \equiv i\left(f_{0}, 0\right)(\bmod 2)$ for $n \geq 1$.

If $i\left(f_{0}, 0\right)=1$ then there exists $p \in \mathbb{Z}$ such that $i\left(f_{0}^{n}, 0\right) \in\{1, p\}$ for $n \geq 1$ ([4]). It follows from [12] that $p=0$ and $p=2$ cannot occur as indices of any iteration if $i\left(f_{0}, 0\right)=1$, hence (2) holds.

If $f_{0}$ is orientation reversing then [12, Theorem 4.2] (compare with [3]) ensures that for every $n$ odd $i\left(f_{0}^{n}, 0\right) \in\{-1,0,1\}$ and

which proves (3).

$$
i\left(f_{0}^{n}, 0\right)= \begin{cases}i\left(f_{0}, 0\right) & \text { if } n>0 \\ -i\left(f_{0}, 0\right) & \text { if } n<0\end{cases}
$$

\section{Final Remarks and Open problems}

Through the paper we have studied continuous maps $f:\left(X ; X_{1}, X_{2}\right) \rightarrow\left(X ; X_{1}, X_{2}\right)$ of ENR triplets having compact fixed point set. We saw that the Mayer-Vietoris formula of the fixed point holds in the following situations

(1) If $X$ is compact,

(2) if $X_{0}$ is a trapping set,

(3) if $\operatorname{Fix}(f) \subset \operatorname{int}(C \backslash E)$ for an admissible proper pair,

(4) if $\left(X ; X_{1}, X_{2}\right)=\left(E^{n} ; E_{+}^{n}, E_{-}^{n}\right)$ and

(5) if $\left(X ; X_{1}, X_{2}\right)$ admits a good Euclidean embedding.

However, the general case remains open

Problem 33. Does it Mayer-Vietoris formula (0.1) hold for general self-maps $f:\left(X ; X_{1}, X_{2}\right) \rightarrow$ $\left(X ; X_{1}, X_{2}\right)$ of ENR triplets whose fixed point set is compact?

Let $X$ be an ENR and $f: X \rightarrow X$ be a continuous map having $x_{0}$ as an isolated fixed point for all its iterates. The sequence of fixed point indices $\left(i\left(f^{n}, x_{0}\right)\right)_{n \geq 1}$ must satisfy the following congruences, known as Dold relations [9]:

$$
\sum_{k \mid n} \mu(n / k) i\left(f^{k}, x_{0}\right) \equiv 0 \quad(\bmod n),
$$

where $\mu$ denotes the arithmetic Möbius function which assigns to each natural number $n$ a value $-1,0$, or 1 depending on its prime decomposition. In particular, $\mu(n)=0$ if $n$ has a repeated factor in its prime decomposition and $\mu(n)=(-1)^{s}$ otherwise, where $s$ is the number of prime factors in its prime decomposition.

Problem 34. Determine which integer sequences $\left(a_{k}\right)_{k \geq 1}$ and $\left(b_{k}\right)_{k \geq 1}$ satisfying Dold relations can be realized in such a way that there exists a homeomorphism $f: \bar{E}_{+}^{n} \rightarrow E_{+}^{n}$ such that 0 is an isolated fixed point for all its iterates and for each $k \geq 1$

$$
a_{k}=i\left(f^{k}, 0\right) \quad \text { and } \quad b_{k}=i\left(f_{0}^{k}, 0\right) .
$$

Consider also the analogous question in the smooth category. [13]

Problem 34 for odd iterates was recently solved by Graff and Jezierski in the smooth category

The solution of the following problem could find some applications in mathematical ecology.

Problem 35. Suppose that $f:\left(E_{+}^{n}, E_{0}^{n}\right) \rightarrow\left(E_{+}^{n}, E_{0}^{n}\right)$ is a continuous map. It is known (see [31]) that if $S \subset E_{0}^{n}$ is an isolated invariant set with respect to $f$ (in the entire space) then

$$
i(f, S)= \begin{cases}i\left(f_{0}, S\right) & \text { if } W^{u}(S, f) \subset E_{0}^{n}, \\ 0 & \text { if } W^{s}(S, f) \subset E_{0}^{n},\end{cases}
$$

Does it remain true if $S \subset E_{0}^{n}$ is an isolated compact set of fixed points of $f$ but not necessarily isolated as an invariant set?

\section{ACKNOWLEDGEMENTS}

The authors would like to express their gratitude to R. Srzednicki and W. Kucharz for inspiring conversations and comments. They also would like to thank the referee for his useful suggestion which have helped to improve the manuscript.

The first author is supported by the FPI grant BES-2013-062675, the research project MTM201563612-P and the mobility grant EEBB-I-16-11119 from the Ministerio de Economía y Competitividad. He is also grateful for the hospitality received during his visit to the Faculty of Mathematics and Computer Science of Jagiellonian University in Krakow. 


\section{REFERENCES}

1. M. Arkowitz, R. F. Brown, The Lefschetz-Hopf theorem and axioms for the Lefschetz number, Fixed Point Theory Appl. 1 (2004), 1-11.

2. C. Bonatti, J. Villadelprat, The index of stable critical points, Topology Appl. 126, (2002), 263-271.

3. M. Bonino, Lefschetz index for orientation reversing planar homeomorphisms, Proc. AMS 130 (2002) (7), 2173-2177.

4. M. Brown, On the fixed point index of iterates of planar homeomorphisms, Proc. AMS 108 (1990) (4), 1109-1114.

5. R. F. Brown, R. E. Greene, H. Schirmer, Fixed points of map extension, in Boju Jiang (Ed.), Topological Fixed Point Theory and Aplications, Lect. Notes in Math. 1411 (1989), pp. 24-45.

6. A. Capietto, B. M. Garay, Saturated invariant sets and the boundary behaviour of differential Systems, J. Math. Anal. Appl. 176, (1993), 166-181.

7. E. N. Dancer, R. Ortega, The index of Lyapunov stable fixed points, J. Dyn. Diff. Eq. 6 (1994), 631-637.

8. A. Dold, Fixed point index and fixed point theorem for Euclidean neighborhood retracts, Topology 4 (1965), $1-8$.

9. A. Dold, Fixed point indices of iterated maps, Invent. Math. 74 (1983), 419-435.

10. F. Dumortier, J. Llibre, J.C. Artés, Qualitative Theory of Planar Differential Systems, Series: Universitext, Springer-Verlag, Berlín, 2006.

11. B. M. Garay, J. Hofbauer, Robust permanence for ecological differential equations, minimax, and discretizations, SIAM. J. Math. Anal. 34 (2003) 1007-1039.

12. G. Graff, P. Nowak-Przygodzki, Fixed point indices of iterations of planar homeomorphisms, Topol. Meth. Nonl. Anal. 22 (2003), 159-166.

13. G. Graff, J. Jezierski, Minimal number of periodic points of smooth boundary-preserving self-maps of simplyconnected manifolds, J. Geom Dedicata (2016) doi:10.1007/s10711-016-0199-4.

14. L. Hernandez-Corbato, F. R. Ruiz del Portal, Fixed point indices of planar continuous maps, Discrete and Cont. Dyn. Sys. 35, (2015), 2979-2995.

15. M. W. Hirsch, Differential Topology, Series: Graduate Texts in Mathematics, vol. 33, Springer-Verlag, NewYork 1994 .

16. J. Hofbauer, Saturated equilibria, permanence, and stability for ecological systems, in "Mathematical Ecology, Proc. Trieste, 1986" (L. J. Gross, T. G. Hallman, and S. A. Levin, Eds.), pp. 625-642, World Scientific.

17. M. Izydorek, S. Rybicki, Z. Szafraniec, A note on the Poincaré-Bendixson index theorem, Kodai Math. J. 19, (1996) (2), 145-156.

18. J. Jezierski, W. Marzantowicz, Homotopy Methods in Topological Fixed and Periodic Points Theory, Series: Topological Fixed Point Theory and Appl., vol. 3, Springer, 2005.

19. B. Jiang, Lectures on Nielsen Fixed Point Theory, Series: Contemporary Mathematics, vol. 14, Amer. Math. Soc., Providence, RI, 1983

20. N. Khamsemanan, R. F. Brown, C. Lee, S. Dhompongsa, Interior fixed points of unit-sphere-preserving Euclidean maps, Fixed Point Th. Appl., 2012/1/183.

21. N. Khamsemanan, R. F. Brown, C. Lee, S. Dhompongsa, A fixed point theorem for smooth extension maps, Fixed Point Th. Appl. , 2014/1/197.

22. P. Le Calvez, J.-C. Yoccoz, Un théoreme d'indice pour les homéomorphisms du plan au voisinage d'un point fixe, Ann. Math. 146, (1997), 241-293.

23. J.M. Lee, Introduction to Smooth Manifolds, Graduate Texts in Mathematics, 218, Springer-Verlag, New York 2003.

24. J.W. Milnor, Topology from the Differentiable Viewpoint, Princeton University Press, 1997.

25. P. Pelikan, E. E. Slaminka, A bound for the fixed point index of an area-preserving homeomorphism of two-manifolds, Ergod. Th. Dyn. Syst. 7 (1987), 463-479.

26. A. Ruiz-Herrera, Permanence of two species and fixed point index, Nonlinear Anal. 74 (2011) 146153

27. F. R. Ruiz del Portal, Planar isolated and stable fixed points have index = 1, J. Diff. Eq. 199, (2004), $179-188$.

28. F. R. Ruiz del Portal, J. M. Salazar, Fixed point index o,f iterations of local homeomorphism of the plane: a Conley index approach, Topology 41 (2002), 1199-1212.

29. F. R. Ruiz del Portal, J. M. Salazar, Indices of the iterates of $\mathbb{R}^{3}$-homeomorphisms at Lyapunov stable fixed points, I. Diff. Eq. $\mathbf{2 4 4}$ (2008), 1141-1156.

30. R. Srzednicki, Generalized Lefschetz Theorem and Fixed Point Index Formula, Topol. Appl. 81, (1997), 207-224.

31. A. Szymczak, K. Wójcik, P. Zgliczyński, On the discrete Conley index in the invariant subspace, Topol. Appl. 87, (1998), 105-115.

32. E. H. Spanier, Algebraic topology, McGraw-Hill Book Co., 1966.

33. K. Wójcik, Conley index and permanence in dynamical systems, Top. Meth. Nonl. Anal. 12 (1998), $153-158$.

34. K. Wójcik, An attraction result and an index theorem for a continuous flows on $\mathbb{R}^{n} \times[0,+\infty)$, Ann. Polon. Math. 65 (1997), 203-211.

Facultad de C.C. Matemáticas, Universidad Complutense de Madrid, Plaza de las Ciencias 3, 28040 Madrid, Spain Department of Mathematics and Computer Science, Jagiellonian University, Lojasiewicza 6, 30-348 Kraków, POLAND

E-mail address: hbarge@ucm.es

E-mail address: Klaudiusz.Wojcik@uj.edu.pl 